Document downloaded from:

http://hdl.handle.net/10251/107394

This paper must be cited as:

Vega, J.; Andrio, A.; Lemus, AA.; Del Castillo, LF.; Compañ Moreno, V. (2017). Conductivity study of Zeolitic Imidazolate Frameworks, Tetrabutylammonium hydroxide doped with Zeolitic Imidazolate Frameworks, and mixed matrix membranes of

Polyetherimide/Tetrabutylammonium hydroxide doped with Zeolitic Imidazolate Frameworks for proton conducting applications. Electrochimica Acta. 258:153-166.

doi:10.1016/j.electacta.2017.10.095

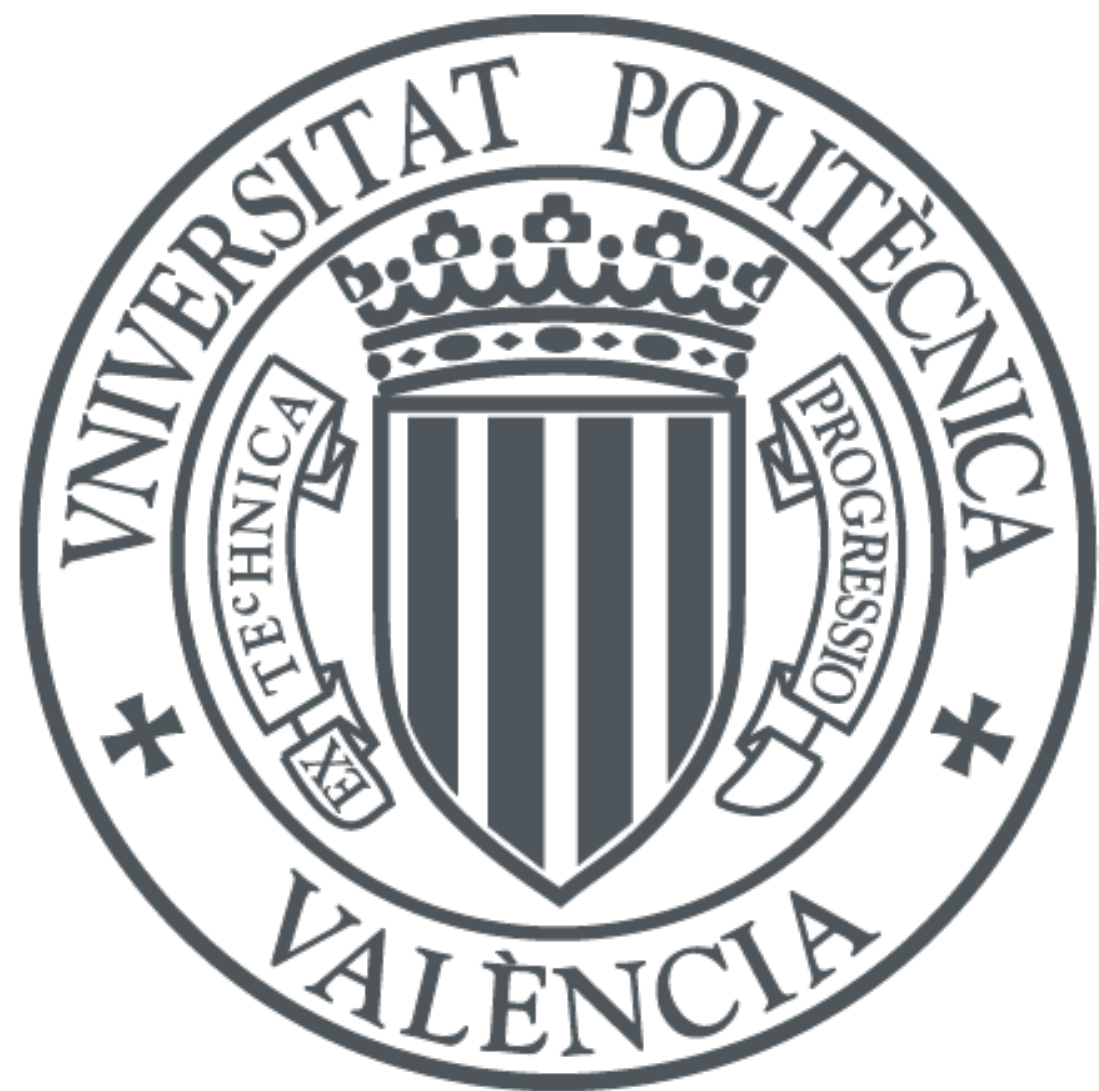

The final publication is available at

https://doi.org/10.1016/j.electacta.2017.10.095

Copyright Elsevier

Additional Information 


\section{Conductivity study of Zeolitic Imidazolate Frameworks, Tetrabutylammonium hydroxide doped with Zeolitic Imidazolate Frameworks, and mixed matrix}

membranes of Polyetherimide/Tetrabutylammonium hydroxide doped with Zeolitic Imidazolate Frameworks for proton conducting applications

J. Vega

A. Andrio

A.A. Lemus

L.F. del Castillo

V. Compañ ${ }^{\mathrm{d}, *}$

vicommo@ter.upv.es

${ }^{a}$ Centro de Investigación en Ciencia Aplicada y Tecnología Avanzada, Unidad Legaría, Instituto Politécnico Nacional, Legaria Núm. 694, Col. Irrigación, Miguel Hidalgo, C.P. 11500 Cd, Mexico

${ }^{\mathbf{b}}$ Departamento de Física aplicada, Universitat Jaume I, 12080, Castellón, Spain

'Departamento de Polímeros, Instituto de Investigaciones en Materiales, Universidad Nacional Autónoma de México (UNAM), Ciudad Universitaria, Apartado Postal 70-360, Coyoacán, 04510, Mexico

dDepartamento de Termodinámica Aplicada, Escuela Técnica Superior de Ingenieros Industriales (ETSII), Universidad Politécnica de Valencia, Campus de Vera s/n, 46020, Valencia, Spain

${ }^{*}$ Corresponding author

Abstract

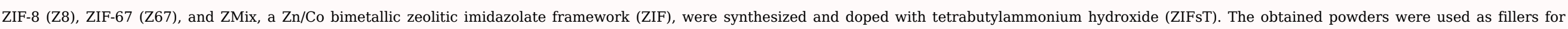

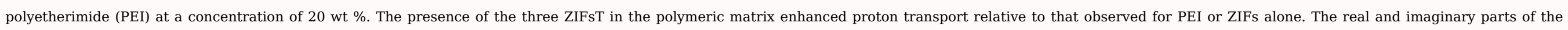

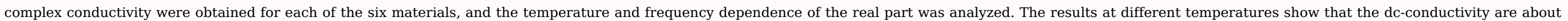

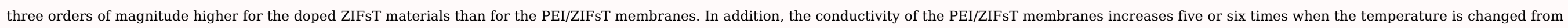

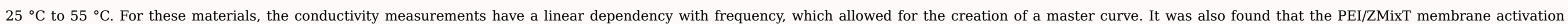

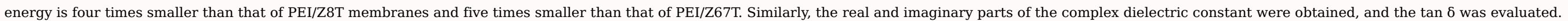

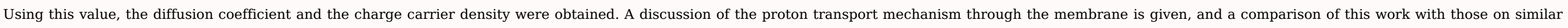
electrolyte membranes is included.

Keywords: Zeolitic Imidazolate Frameworks; Mixed Matrix Membranes; conductivity; PEI/ZIFsT membranes

\section{Introduction}

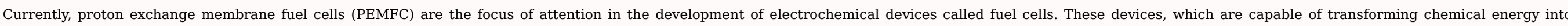

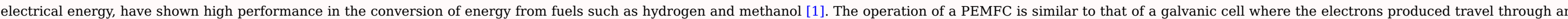

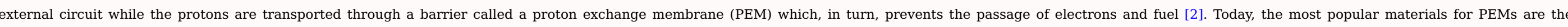

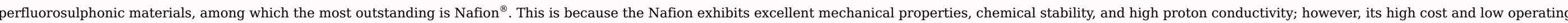

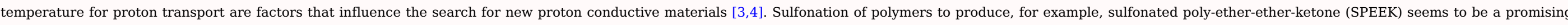

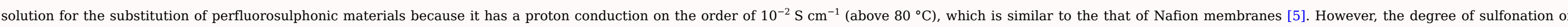




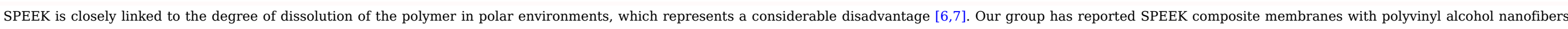

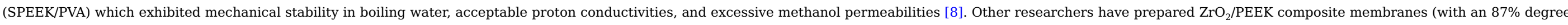

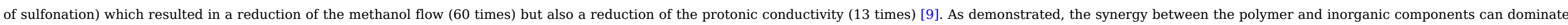

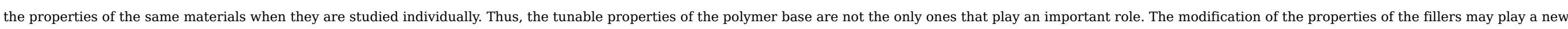

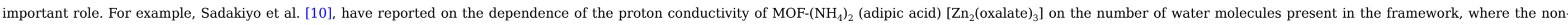
hydrated sample had a very low conductivity whereas the dihydrate and trihydrate samples exhibited conductivities of $7 \times 10^{-5} \mathrm{~S} \mathrm{~cm}^{-1} \mathrm{and}_{8 \times 10^{-3} \mathrm{~S} \mathrm{~cm}}^{-1}$, respectively.

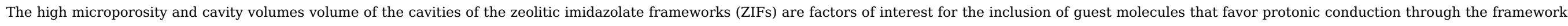

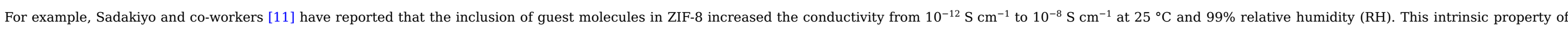
ZIFs ensures that these materials will have high specific chemical reactivity, flexibility, and processability to make membranes oriented toward protonic transport with huge potential for energy applications.

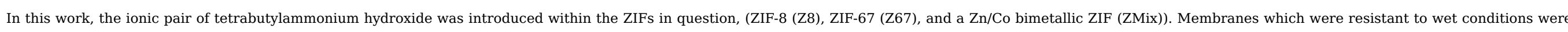

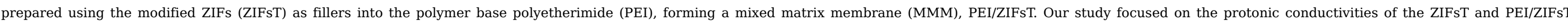

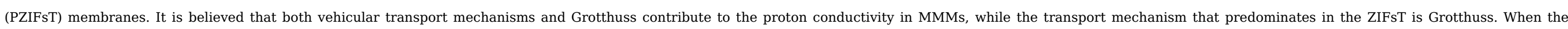

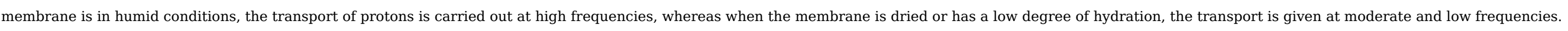

\section{Experimental}

\subsection{Chemical reagents}

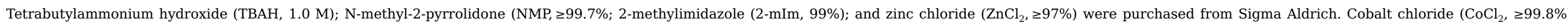
and sodium formate $\left(\mathrm{HCO}_{2} \mathrm{Na}, \geq 99 \%\right)$ were purchased from Baker Analyzed and Reasol respectively.

\subsection{Synthesis of ZIFs (Z8, Z67, and ZMix)}

\subsubsection{Z8 (ZIF-8)}

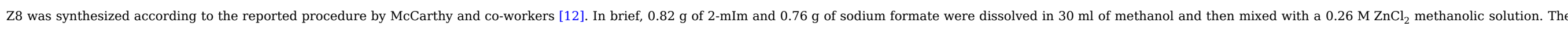
obtained product was washed, centrifuged, and dried under vacuum for $12 \mathrm{~h}$.

\subsubsection{Z67 and ZMix (ZIF-67 and ZIF-Mix)}

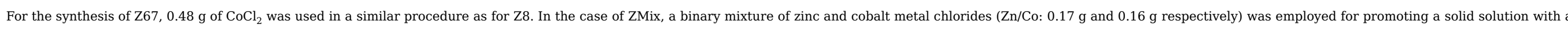
$1: 1$ metal ratio.

\subsubsection{ZIFsT (Z8T, Z67T, and ZMixT)}

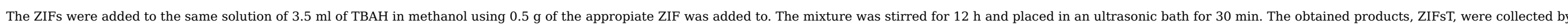
centrifugation, washed with deionized water, and dried under vacuum at $30^{\circ} \mathrm{C}$.

\subsection{PZIFs and PZIFsT Mixed Matrix Membranes (PEI/ZIFs and PEI/ZIFsT)}

\subsubsection{PZIFs, PZIFsT}

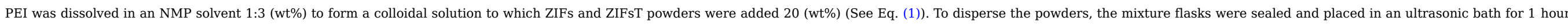

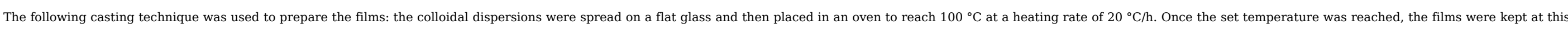

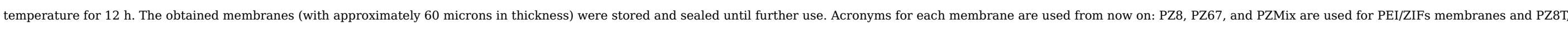


$\%$ weight $\mathrm{ZIF}=\frac{\text { weight } \mathrm{ZIF}}{\text { weight } \mathrm{ZIF}+\text { weight } \mathrm{PEI}} \times 100 \%$

\subsection{Scanning electron microscopy (SEM) study}

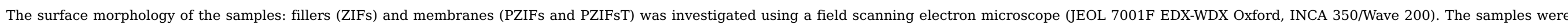
gold coated and conserved before SEM observations.

\subsection{Thermogravimetric analysis}

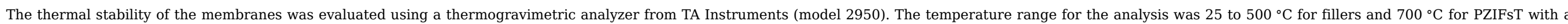
heating rate of $5{ }^{\circ} \mathrm{C} / \mathrm{min}$. The experiments were carried out under nitrogen atmosphere using a flux of $60 \mathrm{ml} / \mathrm{min}$.

\subsection{Infrared spectroscopy (FTIR)}

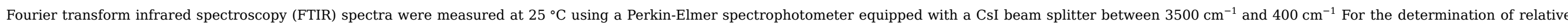

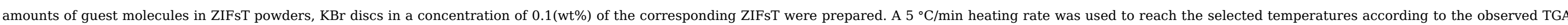
stages. Once the target temperature was reached, it was maintained for $5 \mathrm{~min}$, and then the disc was analyzed by IR.

\subsection{Porosity analysis}

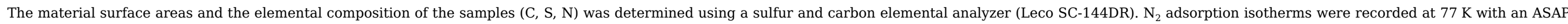

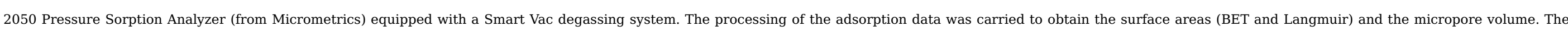
sample was activated under vacuum at $100^{\circ} \mathrm{C}$ for 2 hours before thermogravimetric analysis.

\subsection{Impedance spectroscopy measurements}

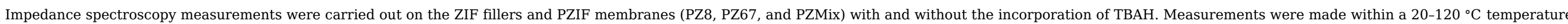

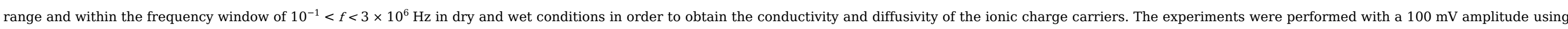

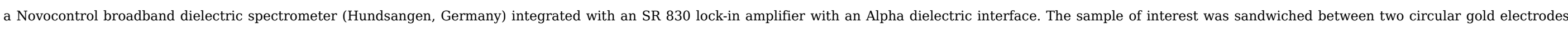

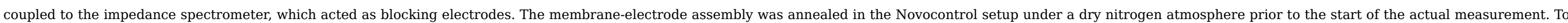

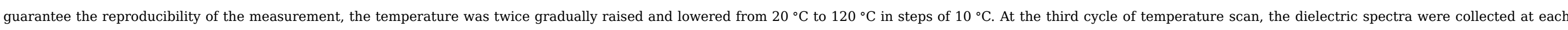
step.

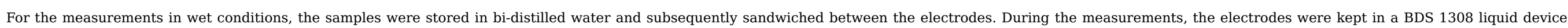

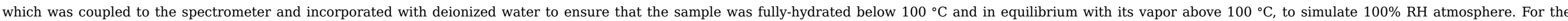
isothermal experiments, the temperature was controlled by a nitrogen jet (QUATRO from Novocontrol) with a temperature error of $0.1 \mathrm{~K}$ during every individual sweep in frequency.

\section{Results and discussion}

\subsection{Scanning electron microscopy analysis}

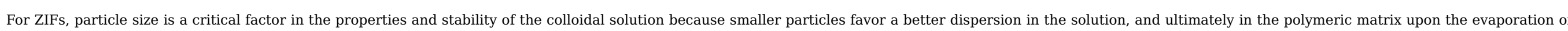

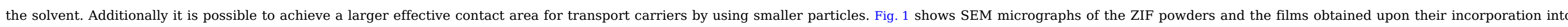

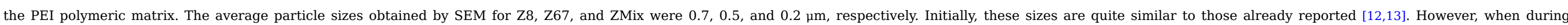




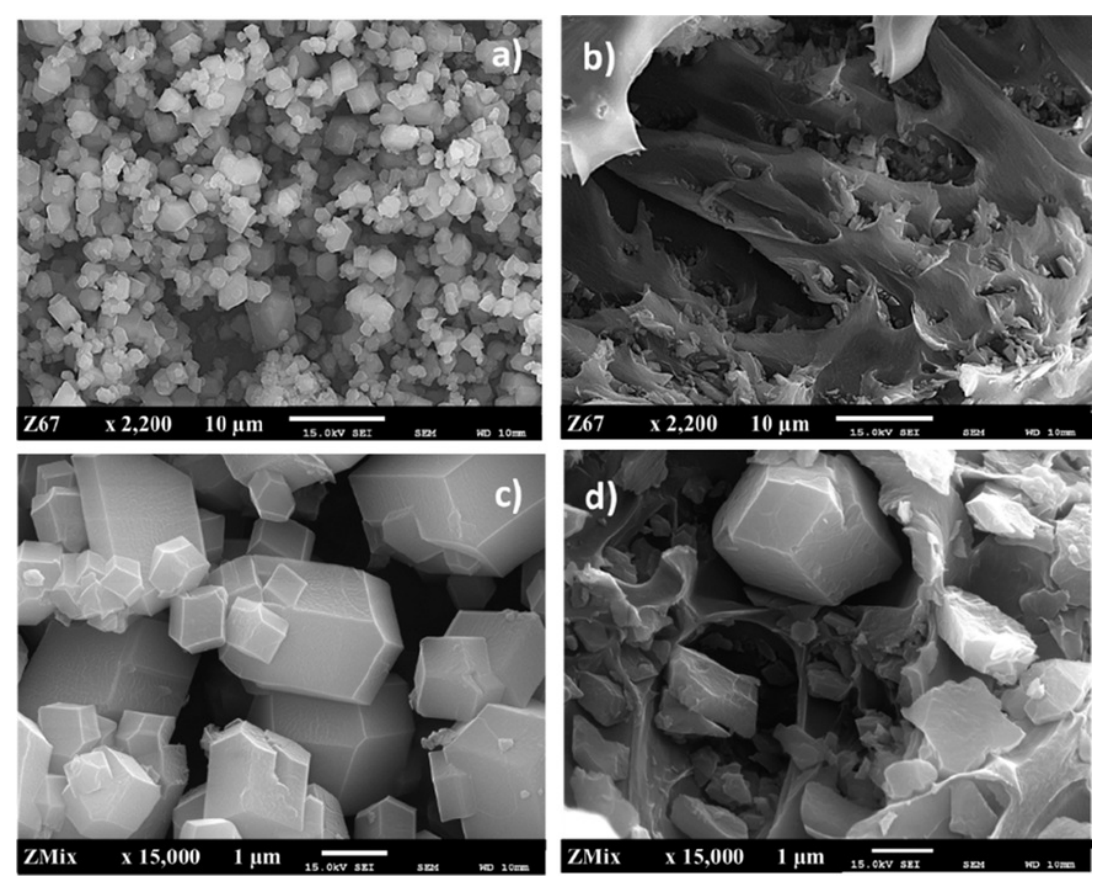

Fig. 1 SEM micrographs of powder materials a) Z67 and c) ZMix; within the PEI. b) PZ67 and d) PZMix, polymeric matrix. alt-text: Fig. 1

\subsection{Analysis of guest molecules in ZIFsT}

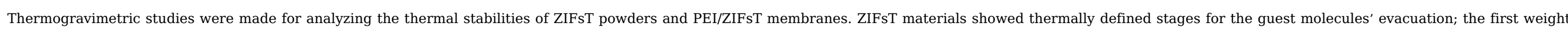

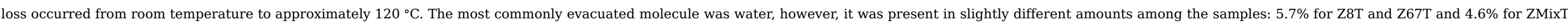

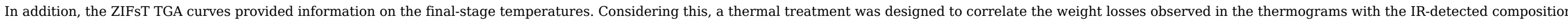

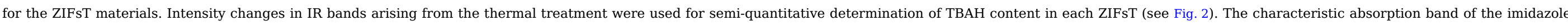

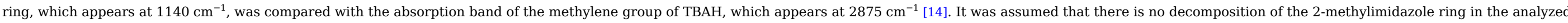
temperature range, therefore, the $1140 \mathrm{~cm}^{-1}$ band was taken as a reference to calculate the relative concentration of methylene groups upon heat treatment. 


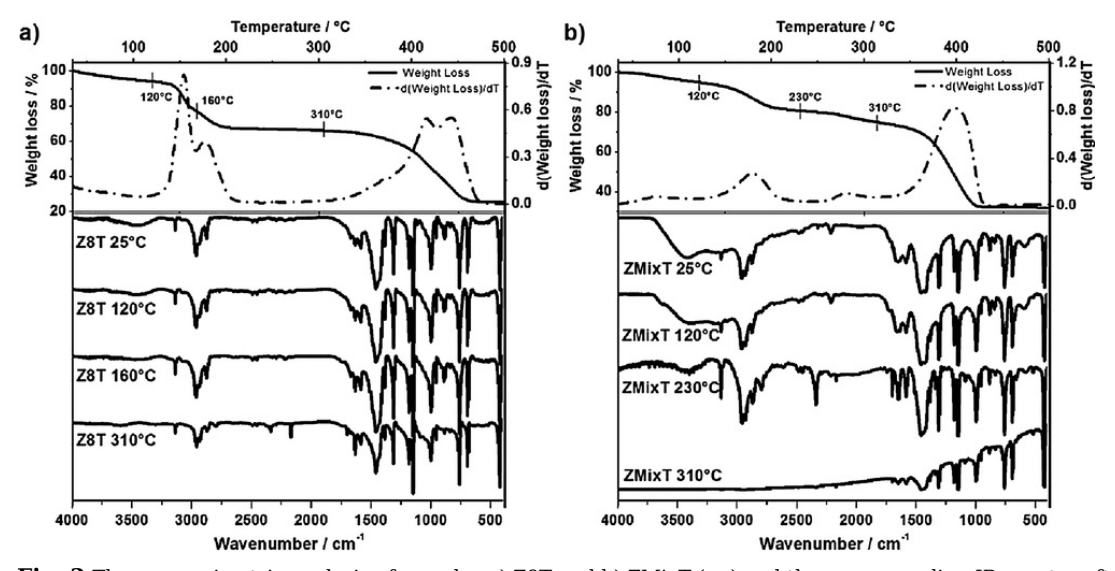

Fig. 2 Thermogravimetric analysis of samples a) Z8T and b) ZMixT (up) and the corresponding IR spectra after controlled thermal treatment (down) for the same materials. alt-text: Fig. 2

The results are summarized in Table 1, where the relative TBAH concentration in ZMixT is more than twice the relative TBAH concentration in Z8T and Z67T.

Table 1 Relative concentration of guest molecules.

alt-text: Table 1

Sample Temperature $/{ }^{\circ} \mathrm{C} \quad$ Imidazole ring band $\left.\left(\left(\mathrm{C}_{4} \mathrm{H}_{5} \mathrm{~N}_{2}\right)_{2} \mathrm{M}\right)^{\mathrm{b}}\right) \quad$ Absorbance/\%a ${ }^{\mathrm{a}} \quad$ Methylene group band (TBAH)

Relative amount of guest moleculesc TBA Abs/12 $\mathrm{CH}_{2}$ units

$\left(\mathrm{C}_{4} \mathrm{H}_{5} \mathrm{~N}_{2}\right)$ Abs/24 rings in a cell unit

\begin{tabular}{|c|c|c|c|c|}
\hline \multirow[t]{4}{*}{ Z8T } & 25 & 81.6 & 20.1 & 0.49 \\
\hline & 120 & 83.5 & 20.3 & 0.49 \\
\hline & 150 & 85.3 & 20.5 & 0.48 \\
\hline & 310 & 73 & 8.9 & 0.24 \\
\hline \multirow[t]{4}{*}{ Z67T } & 25 & 81.6 & 21 & 0.51 \\
\hline & 120 & 82.9 & 21.1 & 0.51 \\
\hline & 230 & 84.8 & 21.4 & 0.50 \\
\hline & 310 & 73.9 & 8.7 & 0.24 \\
\hline \multirow[t]{4}{*}{ ZMixT } & 25 & 96 & 65 & 1.35 \\
\hline & 120 & 97.7 & 62.4 & 1.28 \\
\hline & 230 & 99.3 & 50.9 & 1.03 \\
\hline & 310 & 99.7 & - & - \\
\hline
\end{tabular}

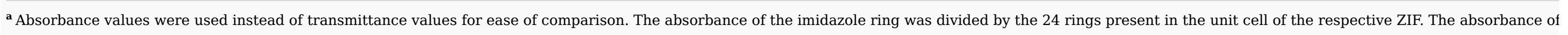
the methylene group was divided by the 12 methylene units present in the tetrabutylammonium cation. 
$\mathbf{b}\left(\mathrm{C}_{4} \mathrm{H}_{5} \mathrm{~N}_{2}\right)_{2} \mathrm{M}$, where $\mathrm{M}=\mathrm{Zn}$, Co, and $\mathrm{Zn} / \mathrm{Co}$ is the empirical formula for each ZIF.

${ }^{\mathbf{c}}$ A nondimensional value: \% Absorbance/\% Absorbance.

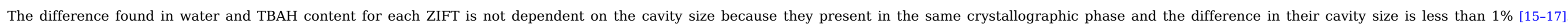

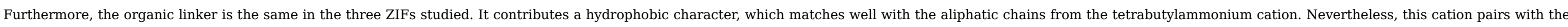

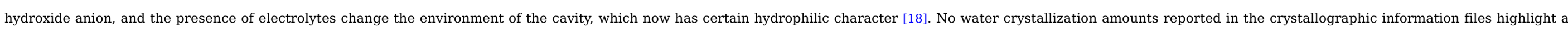

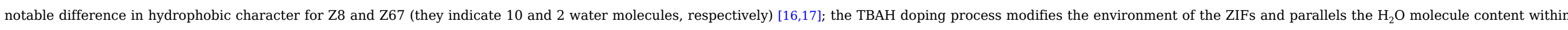

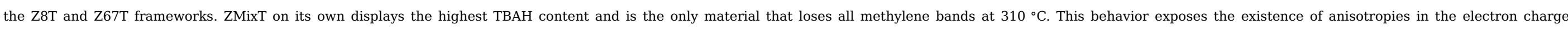

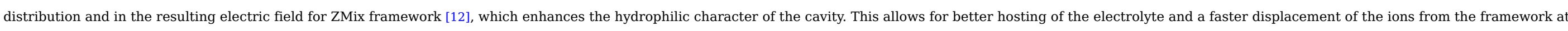
a specific temperature in comparison with Z8 and Z67.

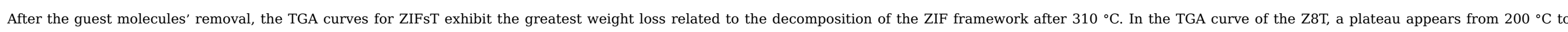

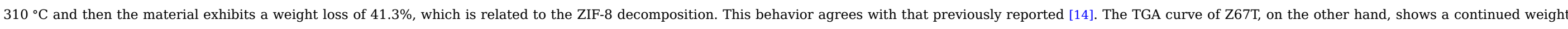

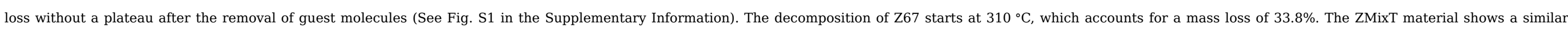
pattern of sustained weight loss once guest molecules leave, but the greatest loss starts at $320^{\circ} \mathrm{C}$ and corresponds to a $41.8 \%$ weight loss, which is related to the ZMix decomposition (Fig. $2 \mathrm{~b}$ ).

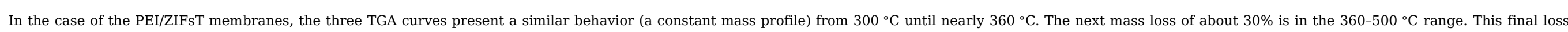
could be related to the decomposition of the ZIFs materials (see Fig. S2). From that point on, the curve follows a profile with a smooth slope which is likely related to the decomposition of the polymer base.

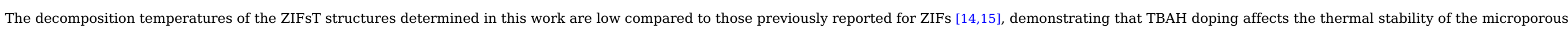
materials.

\subsection{Porosity analysis}

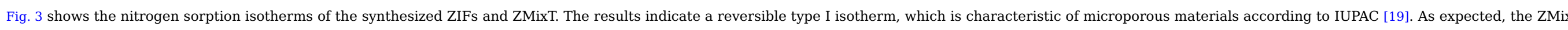

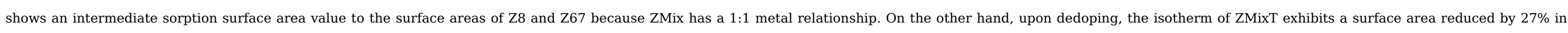
comparison with the ZMix sample.

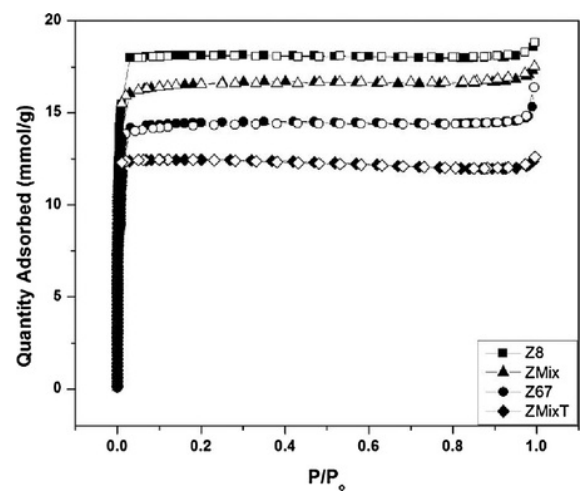

Fig. $3 \mathrm{~N}_{2}$ adsorption/desorption isotherms at $77 \mathrm{~K}$ for ZIFs and ZMixT. alt-text: Fig. 3

Table 2 shows the values obtained in nitrogen sorption/desorption study for the series of materials ZIFs and ZMixT. 
Table 2 Textural properties of ZIFs and ZMixT materials obtained by nitrogen adsorption isotherms. alt-text: Table 2

\begin{tabular}{|c|c|c|c|}
\hline Sample & BET surface area $\left(\mathrm{m}^{2} \mathrm{~g}^{-1}\right)$ & Langmuir surface area $\left(\mathrm{m}^{2} \mathrm{~g}^{-1}\right)$ & Micropore volume $\left(\mathrm{cm}^{3} \mathrm{~g}^{-1}\right)$ \\
\hline Z8 & 1279.4 & 1772.1 & 0.62 \\
\hline ZMix & 1179.7 & 1634.3 & 0.53 \\
\hline $\mathrm{Z} 67$ & 1024.1 & 1420.1 & 0.48 \\
\hline ZMixT & 857.9 & 1214.7 & 0.42 \\
\hline
\end{tabular}

\subsection{Impedance spectroscopy results}

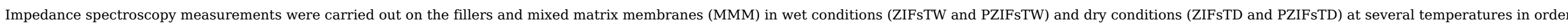

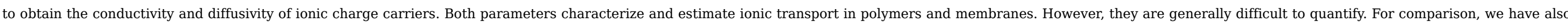

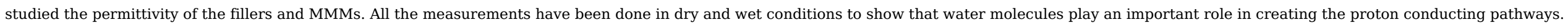

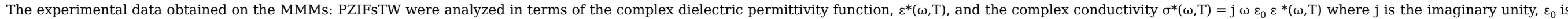
the vacuum permittivity and $\omega$ the angular frequency of the applied electric field $(\omega=2 \pi f)$.

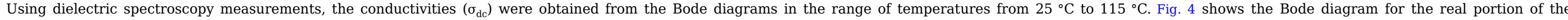
conductivity of the fillers, Z8TW (Fig. 4a), Z67TW (Fig. 4b), and ZMixTW (Fig. 4C), respectively.

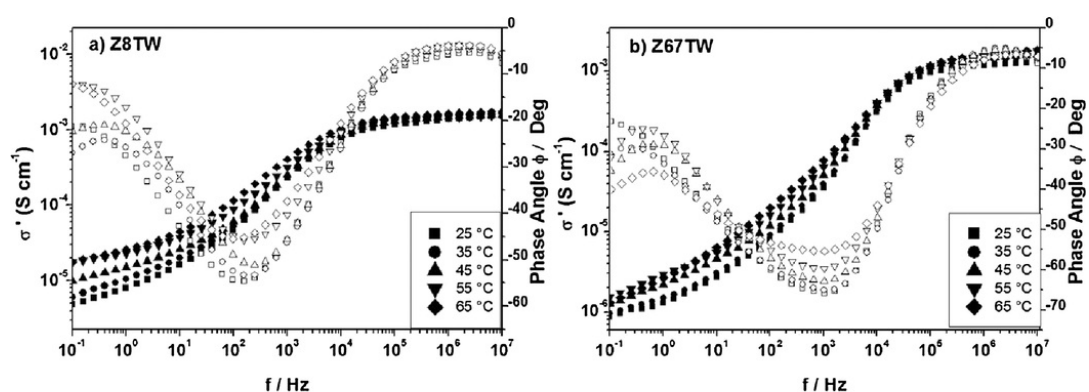

$\mathrm{f} / \mathrm{Hz}$

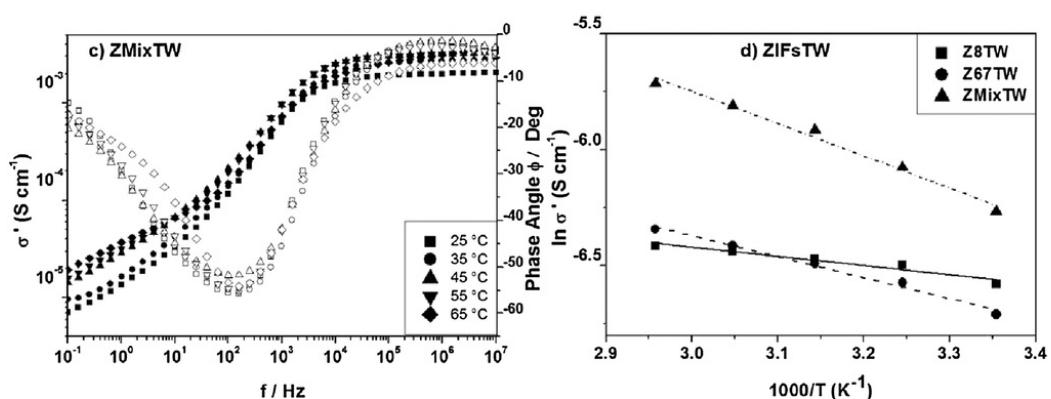

Fig. 4 Bode diagrams for the samples: a) Z8TW, b) Z67TW, c) ZMixTW in the range of temperature between 25 and $65{ }^{\circ} \mathrm{C}$. d) Arrhenius plot for the conductivity of the ZIFsTW.

alt-text: Fig. 4

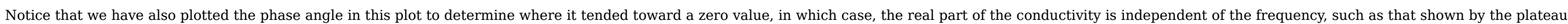




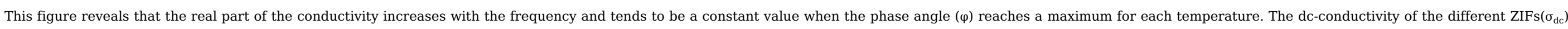

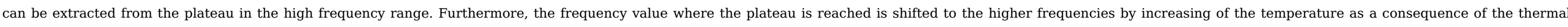

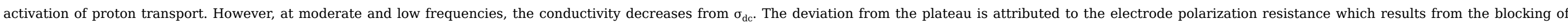
charge carriers at the electrodes [20,21].

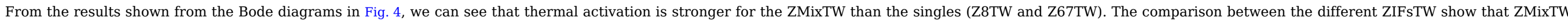

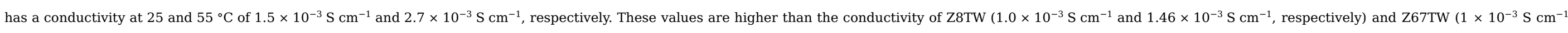
and $1.50 \times 10^{-3} \mathrm{~S} \mathrm{~cm}^{-1}$, respectively)

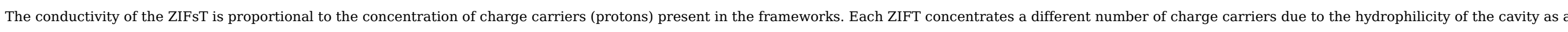

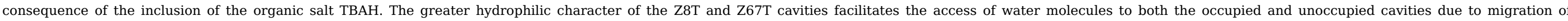

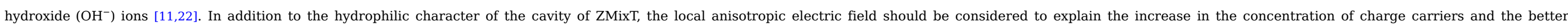
conductivity of ZMixT. The saturated experimental condition under which the conductivity analyses are carried out is the source of the increase in charge carriers.

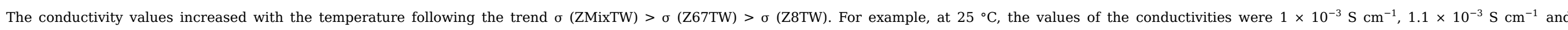

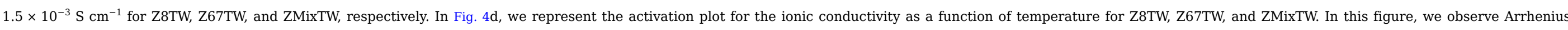

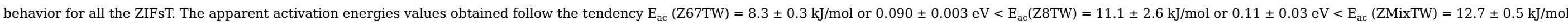
or $0.130 \pm 0.005 \mathrm{eV}$. These values are lower than those found for the activation energy for the conductivity of MOF-based proton conductors [22].

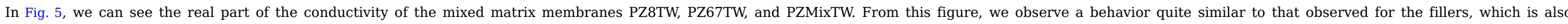

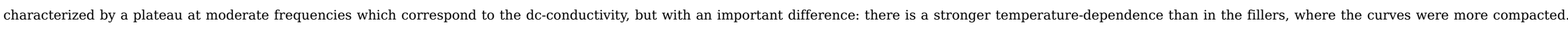

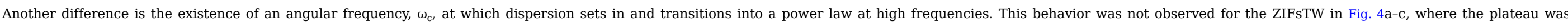
prolonged into the range of high frequencies.

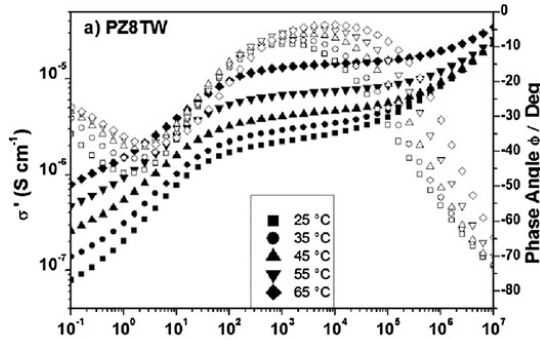

$\mathrm{f} \mathrm{Hz}$

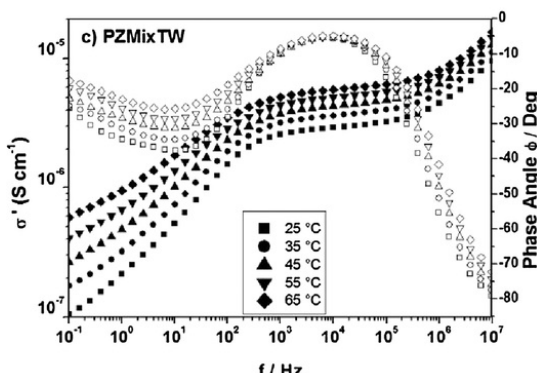

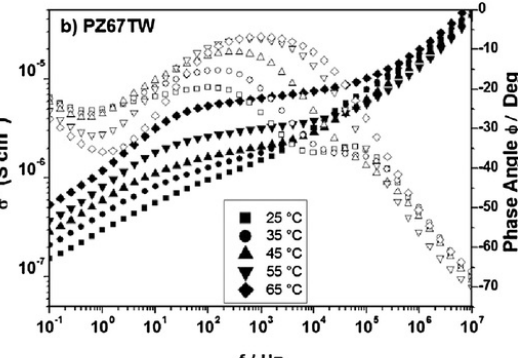

$\mathrm{f} / \mathrm{Hz}$

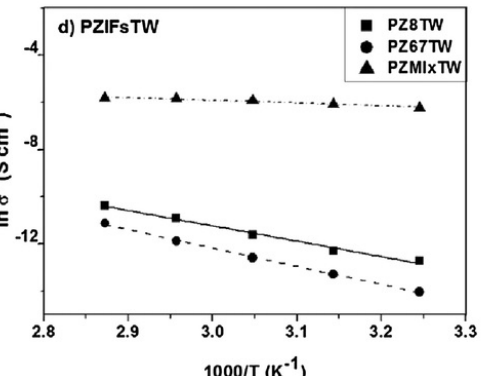

Fig. 5 Bode diagrams for the PZIFsTW samples a) PZ8TW, b) PZ67TW, and c) PZMixTW in the range of temperature between 25 and $65^{\circ} \mathrm{C}$. d) Arrhenius plot of conductivity as a function of inverse temperature of PZIFsTW. 


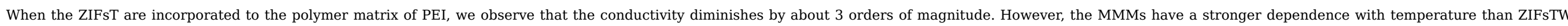

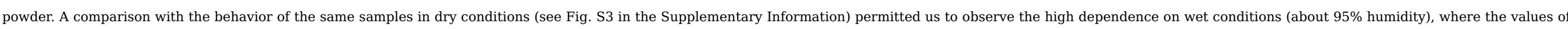

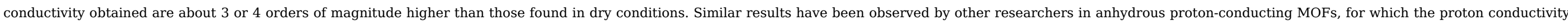

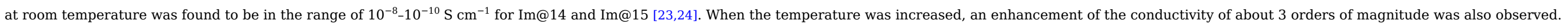

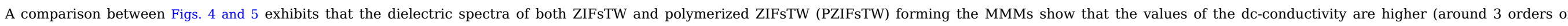

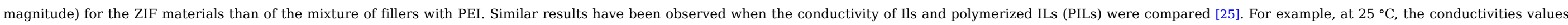

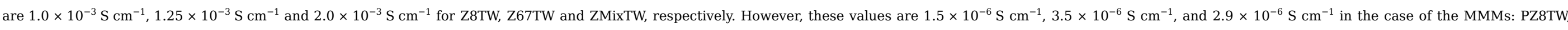

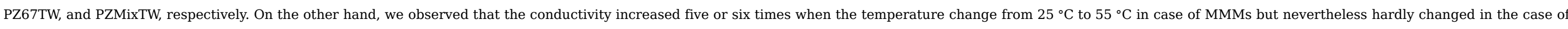

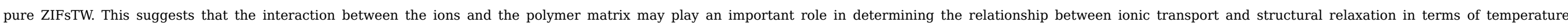
dependence as the fillers are incorporated within the polymeric matrix.

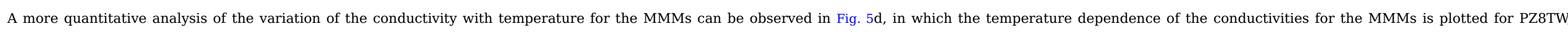

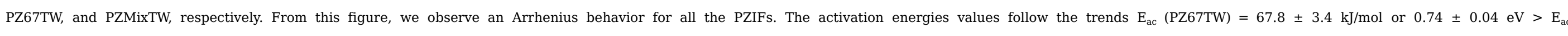
$($ PZ8TW $)=57.1 \pm 8.1 \mathrm{~kJ} / \mathrm{mol}$ or $0.59 \pm 0.08 \mathrm{eV}>\mathrm{E}_{\mathrm{ac}}($ PZMixTW $)=13.5 \pm 0.4 \mathrm{~kJ} / \mathrm{mol}$ or $0.14 \pm 0.03 \mathrm{eV}$.

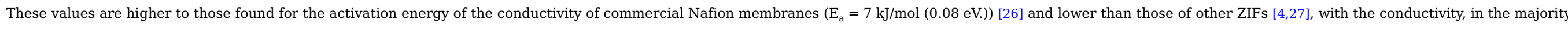
of cases, being higher than or at least similar to $\left(\mathrm{NH}_{4}\right)_{2}$ (adp) $\left(\mathrm{Zn}_{2}(\mathrm{ox})_{3} \cdot 3 \mathrm{H}_{2} \mathrm{O}\right.$ prepared by the isothermal method [10].

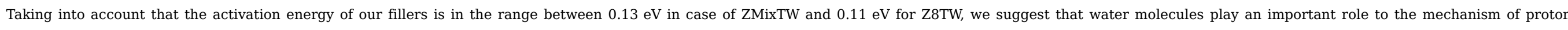

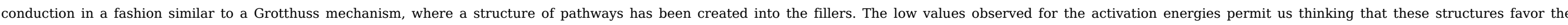

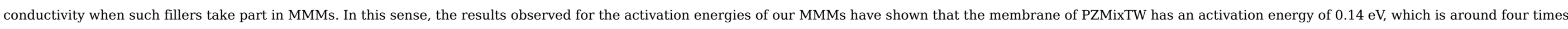
smaller than PZ8TW and five times than PZ67TW, respectively. This value is even smaller than that found for histamine (His@14), for which a value of 0.25 eV was estimated [4].

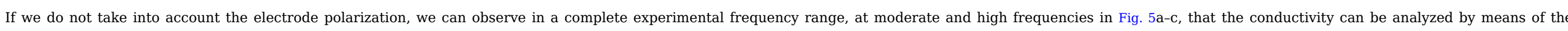

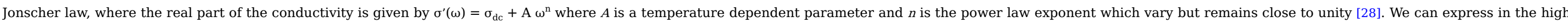
frequency region that $\sigma_{\mathrm{ac}}=\mathrm{A} \omega^{\mathrm{n}}$. In this region, the dielectric permittivity is nearly independent of the frequency and tends to be a plateau, as shown in Fig. S4 for the samples Z8TW and PZ8TW.

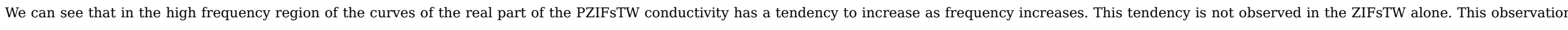

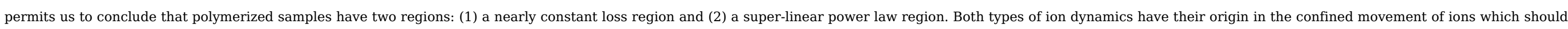

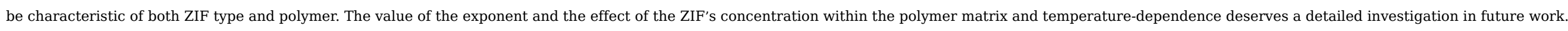

On the other hand, the plateau in the real part of the permittivity is not observed in the case of the ZIFsTW. This is in correspondence with the non-appearance of power-law behavior in case of ZIFsTW alone.

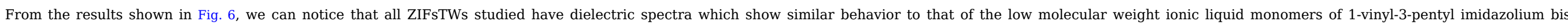

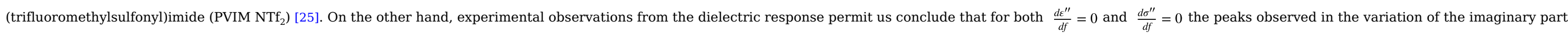
of the conductivity and permittivity appear at the same position on the frequency axes. 

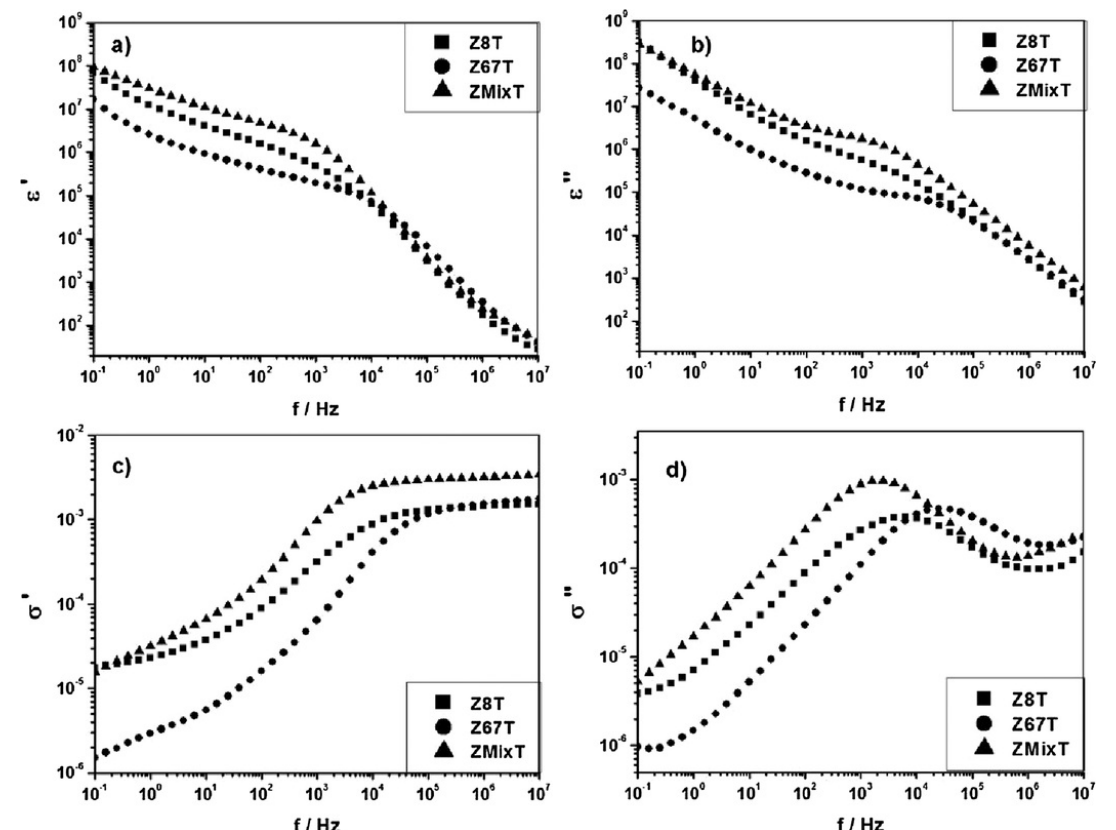

Fig. 6 Double logarithmic plot of the real and imaginary permittivity and conductivity in $\mathrm{S} \mathrm{cm}^{-1}\left(\varepsilon^{\prime}, \varepsilon^{\prime \prime}, \sigma^{\prime}\right.$ and $\left.\sigma^{\prime \prime}\right)$ versus frequency for Z8TW, Z67TW, and ZMixTW at $55^{\circ} \mathrm{C}$

alt-text: Fig. 6

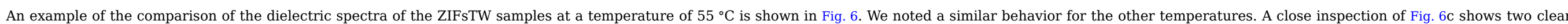

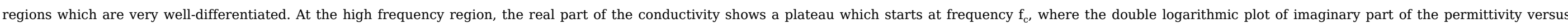

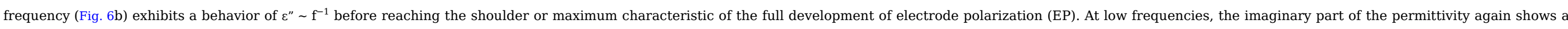

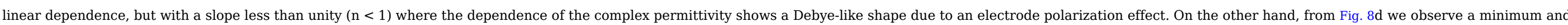
a maximum in $\sigma$ " for all the samples which define the "onset" and the "full development" of electrode polarization (EP) given at frequencies $\mathrm{f}_{\mathrm{ON}}$ and $\mathrm{F}_{\mathrm{MAX}}$, respectively [29].

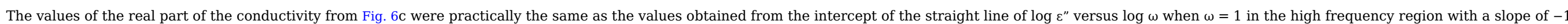

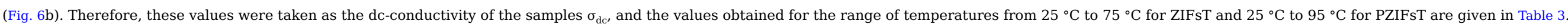

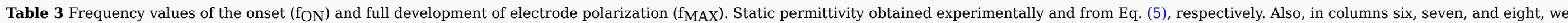
show the dc-conductivity and frequency values obtained at electrode polarization $\left(\mathrm{f}_{\mathrm{EP}}\right)$ and cut-off $\left(\mathrm{f}_{\mathrm{C}}\right)$, which was considered the frequency at which the ac-conductivity is determined.

\section{alt-text: Table 3}

\begin{tabular}{|c|c|c|c|c|c|c|c|}
\hline Temperature $/{ }^{\circ} \mathrm{C}$ & $\mathrm{f}_{\mathrm{MAX}} \times 10^{-3} / \mathrm{Hz}$ & $\mathrm{f}_{\mathrm{ON}} \times 10^{-6 / \mathrm{Hz}}$ & $\varepsilon_{\mathrm{s}}$ (theor) & $\varepsilon_{\mathrm{s}}(\exp )$ & $\sigma_{\mathrm{dc}} \times 10^{3}\left(\mathrm{~S} \mathrm{~cm}^{-1}\right)$ & $\mathrm{f}_{\mathrm{EP}} \times 10^{-3} / \mathrm{Hz}$ & $\mathrm{f}_{\mathrm{c}} \times 10^{-3} / \mathrm{Hz}$ \\
\hline \multicolumn{8}{|l|}{ Z8TW } \\
\hline 25 & 6.3 & 1.0 & 9.9 & - & 1.0 & 6.2 & 30.0 \\
\hline 35 & 6.3 & 1.1 & 9.5 & - & 1.1 & 6.2 & 32.0 \\
\hline 45 & 6.3 & 1.2 & 9.3 & - & 1.3 & 6.2 & 35.0 \\
\hline 55 & 6.3 & 1.3 & 9.1 & - & 1.4 & 6.3 & 37.0 \\
\hline
\end{tabular}




\begin{tabular}{|c|c|c|c|c|c|c|c|}
\hline 65 & 6.3 & 1.4 & 8.8 & - & 1.6 & 6.3 & 39.0 \\
\hline 75 & 6.3 & 1.5 & 8.7 & - & 1.9 & 6.3 & 43.0 \\
\hline \multicolumn{8}{|c|}{ Z67TW } \\
\hline 25 & 25.1 & 1.7 & 16.5 & - & 1.1 & 25.1 & 158.0 \\
\hline 35 & 24.0 & 2.0 & 13.6 & - & 1.2 & 25.2 & 251.0 \\
\hline 45 & 25.1 & 2.2 & 12.4 & - & 1.3 & 25.2 & 398.0 \\
\hline 55 & 25.1 & 2.5 & 10.7 & - & 1.4 & 25.2 & 571.0 \\
\hline 65 & 35.5 & 3.1 & 10.4 & - & 1.6 & 25.2 & 800.0 \\
\hline 75 & 63.1 & 4.3 & 10.8 & - & 1.8 & 25.3 & 1200.0 \\
\hline \multicolumn{8}{|c|}{ ZMixTW } \\
\hline 25 & 2.5 & 6.2 & 17.4 & - & 1.4 & 1.5 & 6.3 \\
\hline 35 & 2.5 & 8.9 & 12.0 & - & 2.0 & 1.5 & 10.0 \\
\hline 45 & 2.6 & 1.0 & 10.9 & - & 2.3 & 1.6 & 15.8 \\
\hline 55 & 2.8 & 1.2 & 8.6 & - & 2.7 & 1.6 & 39.8 \\
\hline 65 & 3.1 & 1.5 & 7.2 & - & 2.9 & 1.6 & 63.1 \\
\hline 75 & 3.3 & 1.7 & 5.8 & - & 3.0 & 1.6 & 100.0 \\
\hline \multicolumn{8}{|c|}{ PZ8TW } \\
\hline 25 & 0.2 & 1.7 & 19.8 & 21.2 & 1.5 & 1.5 & 0.2 \\
\hline 35 & 0.2 & 2.7 & 18.8 & 21.0 & 3.0 & 1.6 & 0.5 \\
\hline 45 & 0.1 & 3.0 & 16.8 & 19.8 & 4.6 & 1.8 & 0.7 \\
\hline 55 & 0.3 & 6.3 & 16.3 & 19.7 & 9.0 & 1.9 & 1.0 \\
\hline 65 & 0.5 & 10.0 & 16.4 & 19.5 & 18.2 & 2.1 & 1.5 \\
\hline 75 & 1.5 & 15.8 & 34.3 & 24.0 & 31.6 & 2.3 & 2.5 \\
\hline 85 & 3.0 & 39.8 & 24.5 & 35.0 & 71.6 & 2.6 & 3.9 \\
\hline 95 & 15.9 & 158.0 & 13.2 & 66.0 & 115.0 & 2.8 & 6.3 \\
\hline \multicolumn{8}{|c|}{ PZ67TW } \\
\hline 25 & - & - & - & - & - & - & - \\
\hline 35 & - & - & - & - & 0.8 & 1.0 & 0.04 \\
\hline 45 & 0.03 & 0.2 & 159.0 & 170.0 & 1.7 & 1.1 & 0.1 \\
\hline 55 & 0.1 & 0.6 & 154.0 & 160.0 & 3.4 & 1.4 & 0.6 \\
\hline
\end{tabular}




\begin{tabular}{|c|c|c|c|c|c|c|c|}
\hline 65 & 0.2 & 1.5 & 125.0 & 140.0 & 6.9 & 1.6 & 2.0 \\
\hline 75 & 0.4 & 3.9 & 66.3 & 82.0 & 14.6 & 1.9 & 6.3 \\
\hline 85 & 1.5 & 15.1 & 44.5 & 62.0 & 35.6 & 2.0 & 25.1 \\
\hline 95 & 3.1 & 31.6 & 42.9 & 50.0 & 75.0 & 2.5 & 50.8 \\
\hline \multicolumn{8}{|c|}{ PZMixTW } \\
\hline 25 & 1.1 & 6.3 & 14.6 & 13.6 & 2.9 & 3.5 & 1.0 \\
\hline 35 & 1.1 & 7.4 & 13.4 & 13.4 & 3.6 & 2.6 & 1.3 \\
\hline 45 & 1.1 & 8.3 & 13.2 & 13.4 & 4.4 & 3.6 & 1.6 \\
\hline 55 & 1.1 & 9.2 & 12.8 & 13.2 & 5.2 & 3.6 & 1.9 \\
\hline 65 & 1.2 & 11.4 & 9.7 & 13.0 & 5.9 & 3.6 & 2.2 \\
\hline 75 & 1.2 & 13.5 & 8.3 & 12.9 & 6.8 & 3.7 & 2.6 \\
\hline 85 & 1.2 & 18.8 & 4.6 & 12.9 & 7.4 & 3.7 & 3.0 \\
\hline 95 & 1.5 & 24.0 & 4.0 & 12.8 & 8.3 & 3.7 & 3.2 \\
\hline
\end{tabular}

\subsubsection{Determination of diffusion coefficient and ion concentration}

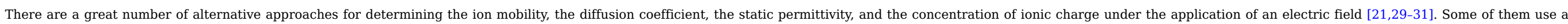

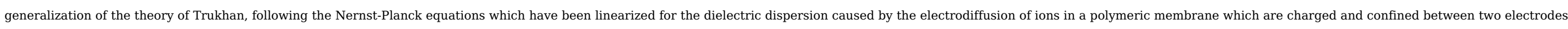
[29-37]. From the analyses of the dielectric spectra of electrode polarizations, we have calculated the ion diffusivity as [33-37]

$D=\frac{\omega_{\max }^{\tan \delta} \cdot L^{2}}{32(\tan \delta)_{\max , \omega}^{3}}$

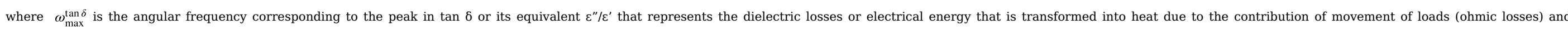

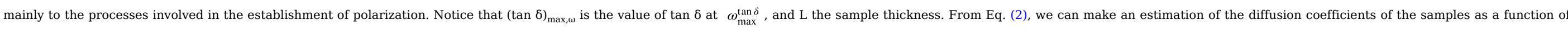
temperature.

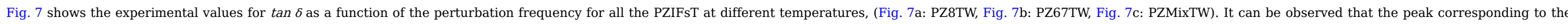

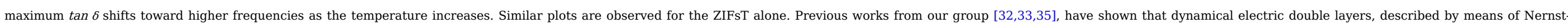

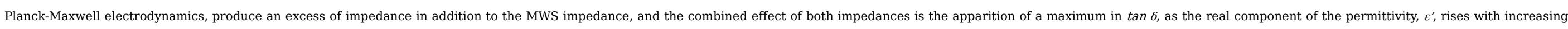
the frequency as a reflection of the capacitance of the macropolarization that, in our case, could be due to $\mathrm{H}^{+}$and $\mathrm{N}(\mathrm{Bu})_{4}{ }^{+}$ions. 


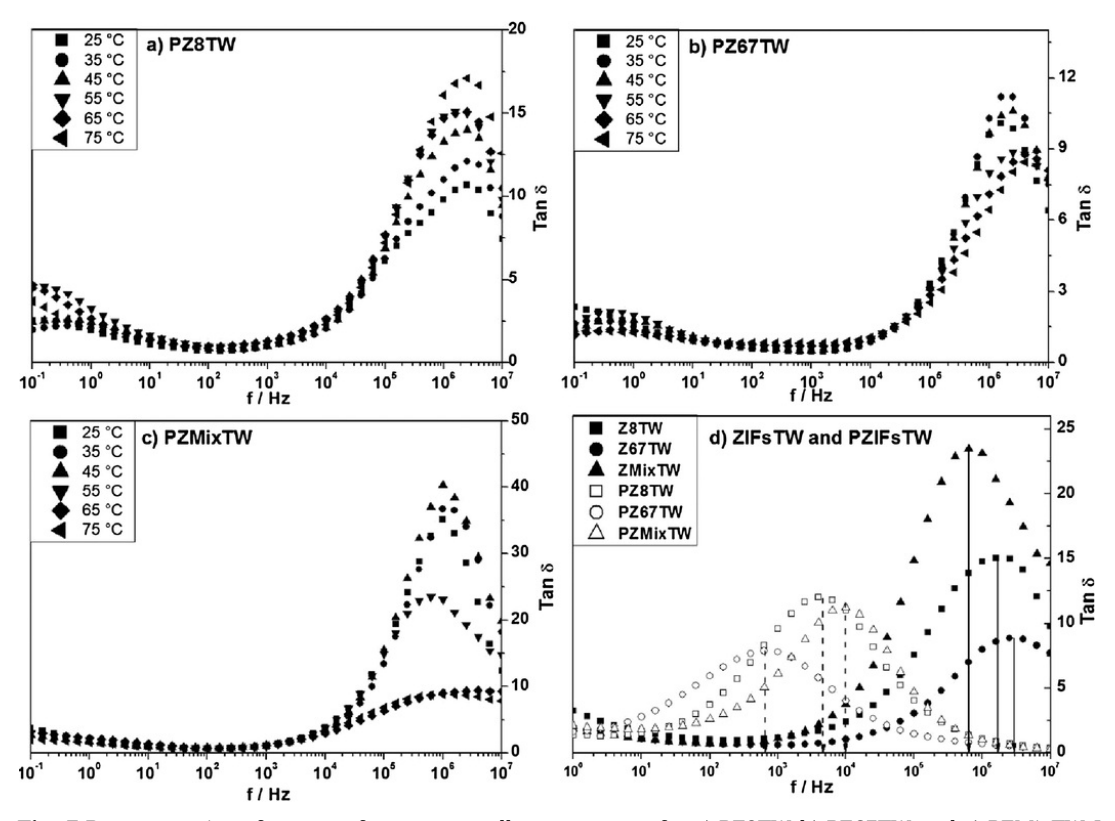

Fig. 7 Representation of tan 6 vs. frequency at all temperatures for a) PZ8TW, b) PZ67TW, and c) PZMixTW. In Figure (d), we plot the tan 6 vs. frequency for all the samples of ZIFsTW and PZIFsTW at $25^{\circ} \mathrm{C}$ for comparison.

alt-text: Fig. 7

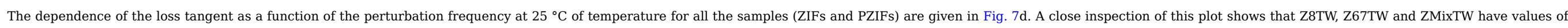
tan $\delta$ higher than the PZIFsTW where the ZIFsTW has been incorporated into the polymeric matrix forming the polymerized ZIFs made up of the MMMs.

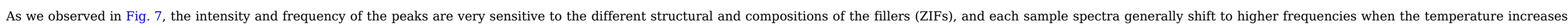

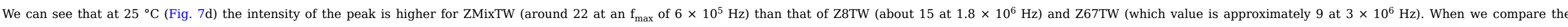

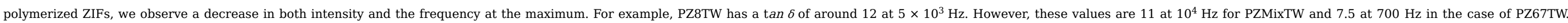
These values can be related to the cross-linking and number of moles of TBAH.

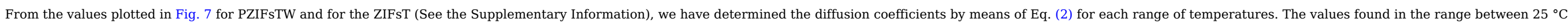
and $75^{\circ} \mathrm{C}$ are plotted in Fig. 8 .

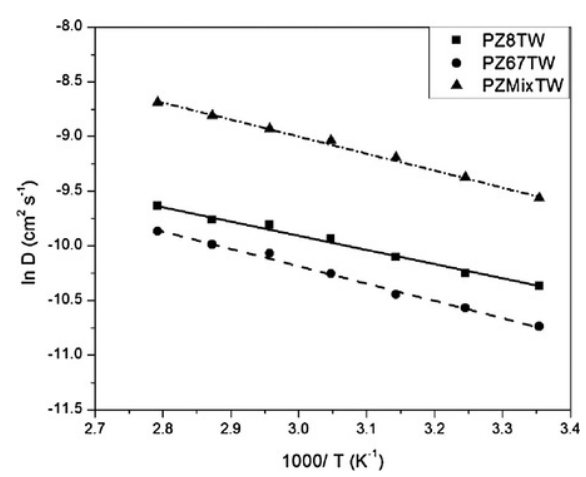

Fig. 8 Arrhenius plot for the diffusion coefficients obtained according to Eq. (2) for the ZIFsTW and PZIFsTW studied. 


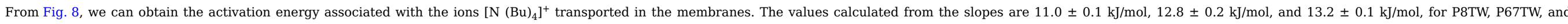

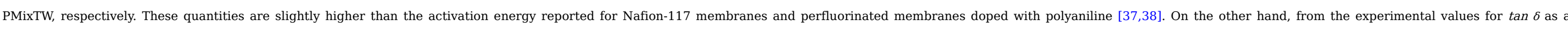

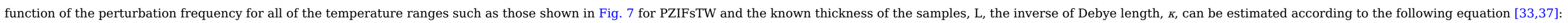

$(\tan \delta)_{\max , \omega} \approx \sqrt{\frac{\kappa L}{8}}$

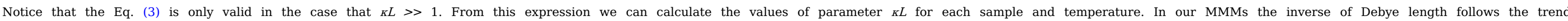
$\mathrm{K}($ PZ8TW $)<\mathrm{K}($ PZ67TW $)<\mathrm{K}\left(\right.$ PZMixTW) at $25^{\circ} \mathrm{C}$, varying from about of $8.5 \times 10^{-7} \mathrm{~m}^{-1}$ for PZ8TW to $2.6 \times 10^{-5} \mathrm{~m}^{-1}$ in the case of PZMixTW.

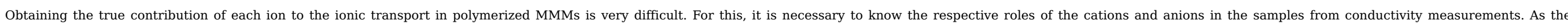

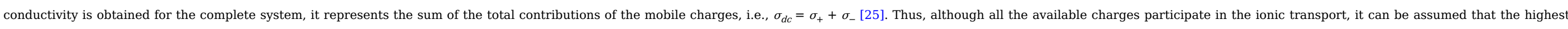

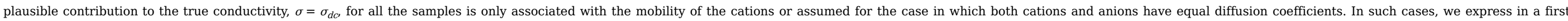
approximation that conductivity and diffusivity are related as $[33,36]$

$\sigma_{d c}=\varepsilon_{s} \varepsilon_{0} \kappa^{2} \frac{D_{+}}{2}$

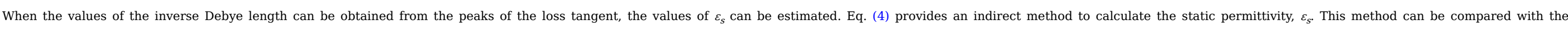
equation of Anatoly Serguei [39], where the permittivity can be expressed in function of the values $f_{O N}$ and $f_{M a x}$ found from the curves of double logarithmic plot of $\sigma$ " versus frequency, as

$\varepsilon_{s}=\frac{\sigma_{d c}}{2 \pi \varepsilon_{0}} \frac{f_{M a x}}{f_{O N}^{2}}$

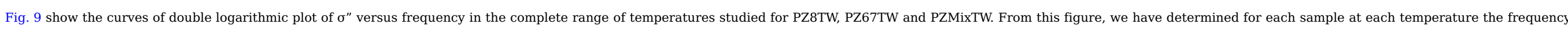

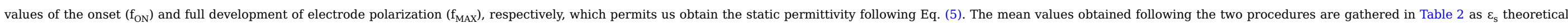

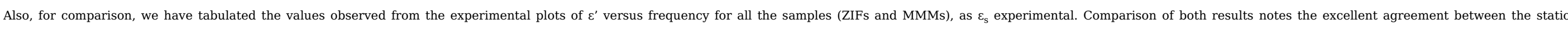

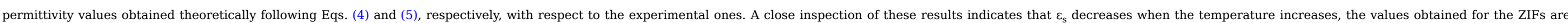
smaller than those of the PZIFs, and both are in good agreement with the values found experimentally. 

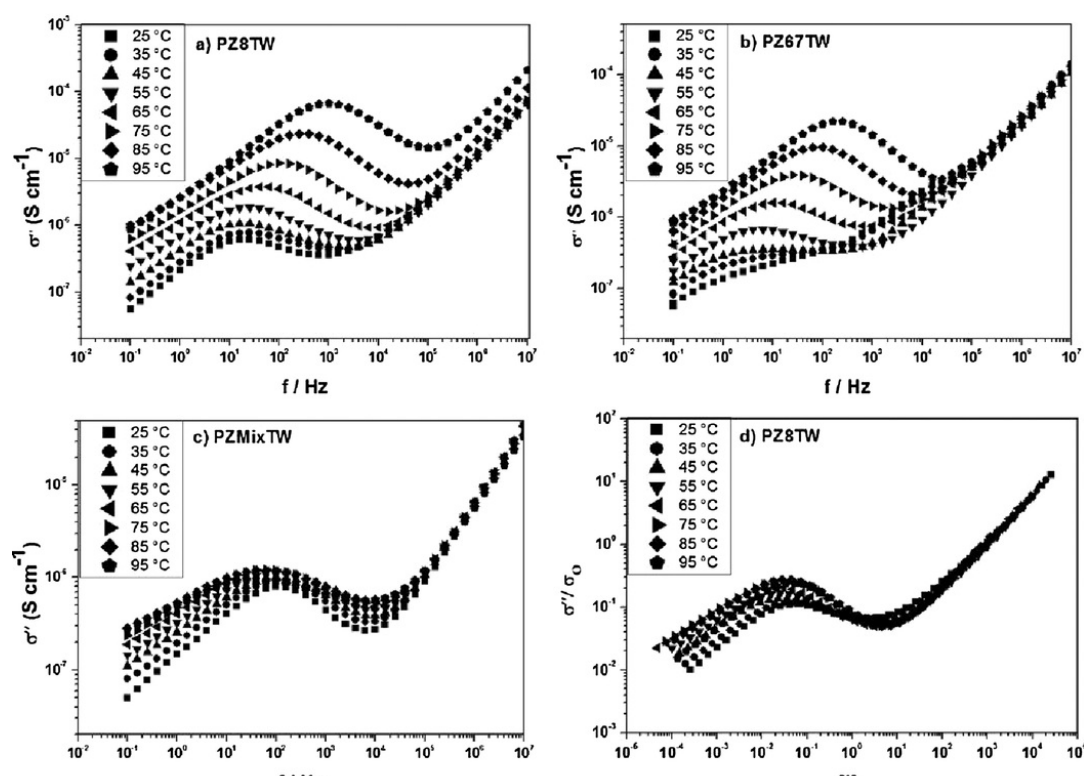

$\mathrm{f} / \mathrm{Hz}$

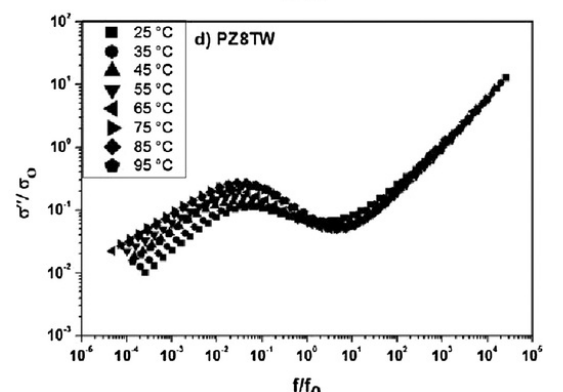

$\mathrm{f} / \mathrm{f}_{0}$

Fig. 9 (a), (b), and (c) are the double logarithmic plots of the imaginary parts of the conductivity vs. frequency for each of the PZIFsTW. (d) Master curve of the normalized plots respect to the imaginary part of the conductivity for PZ8TW. alt-text: Fig. 9

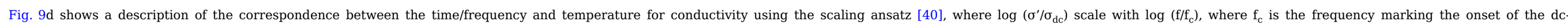

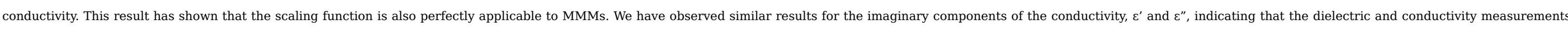

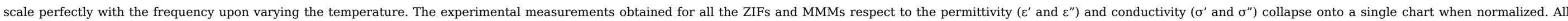
charts collapse into one if scaled with respect to $f_{c}$. This suggests an identical thermal activation of charge transport and electrode polarization.

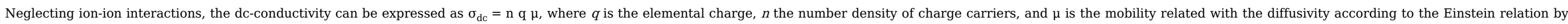
$\mu=q D / k T$, where $D$ is the ionic diffusion coefficient and $k T$ the thermal energy. Finally, the free-ion density can be obtained combining the relations of the dc-conductivity and the ionic mobility as

$n=\frac{k T \cdot \sigma_{d c}}{q^{2} D}$

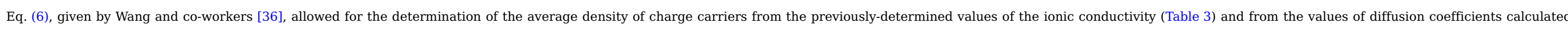

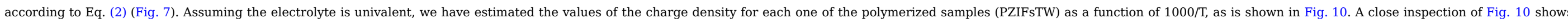

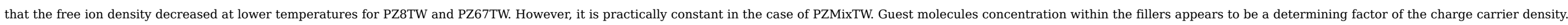
Characteristic local electric anisotropies for each filler governs the different amounts of doping with TBAH through attractive interactions that confine varying amounts of TBAH electrolyte. 


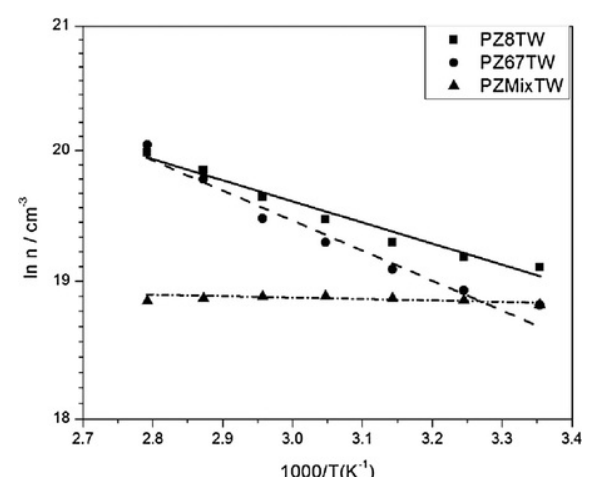

Fig. 10 Temperature dependence of free ion number density (n) for the samples PZIFTW. These values have been calculated from the electrode polarization effect using the generalized Trukhan model described previously in the literature [29,36].

alt-text: Fig. 10

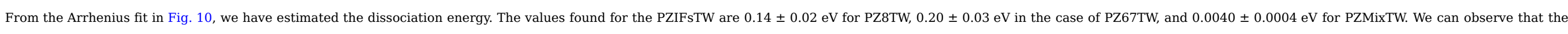

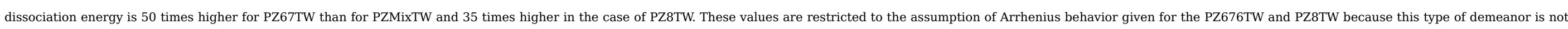
clear for all the range of temperatures studied.

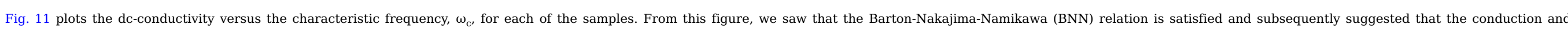

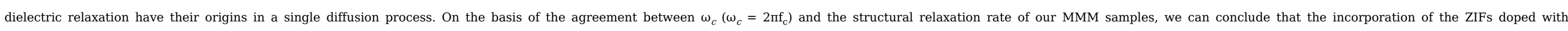

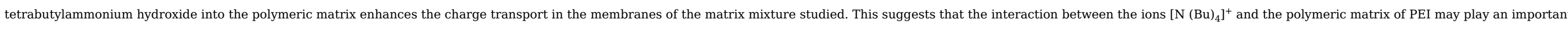

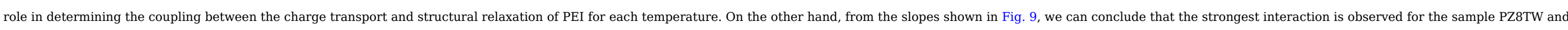
the trends follow the sequence PZ8TW > PZMixTW > PZ67TW.

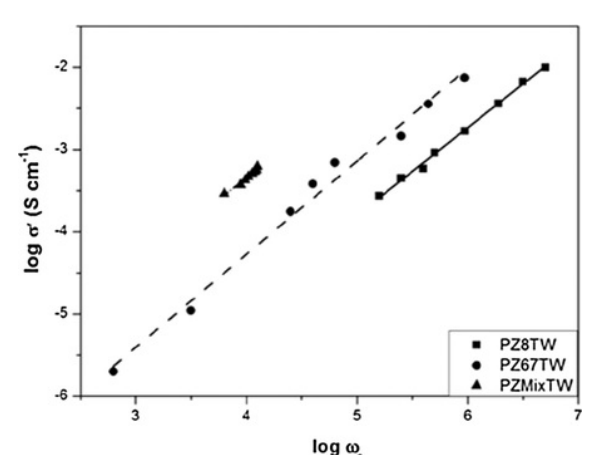

Fig. 11 Dc-conductivity versus the characteristic rates of charge transport $\omega_{\mathrm{c}}$ for the different PZIFsTW.

\section{Conclusions}

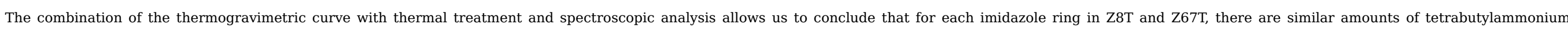
hydroxide $\left[\left(\mathrm{C}_{4} \mathrm{H}_{9}\right)_{4} \mathrm{~N}^{+} \mathrm{OH}^{-}\right]$entities and water molecules while there is more than twofold that amount of the same entity in ZMixT.

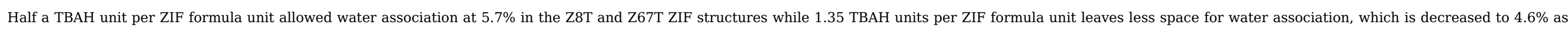

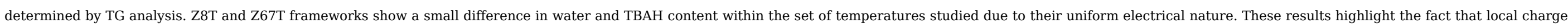




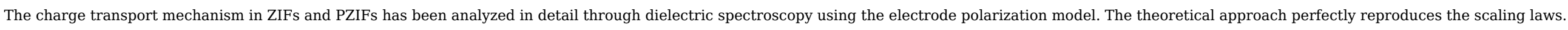

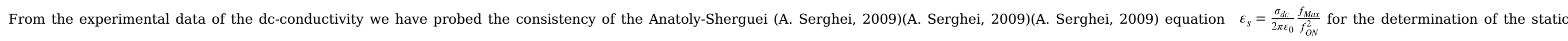
permittivity for Z8TW, Z67TW, ZMixTW, and its MMM obtained on polyether imide films.

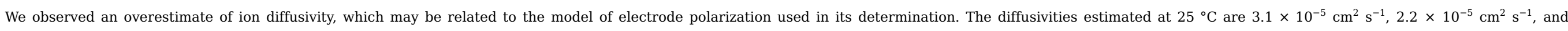
$7.0 \times 10^{-5} \mathrm{~cm}^{2} \mathrm{~s}^{-1}$ for PZ8TW, PZ67TW, and PZMixTW, respectively.

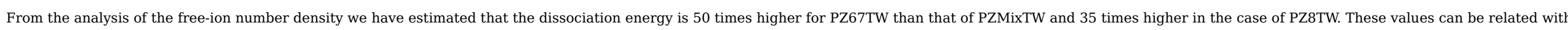
an overestimation of ion diffusivity. These results can be attributed to the electrode polarization analysis of ionic mobility.

\section{Acknowledgements}

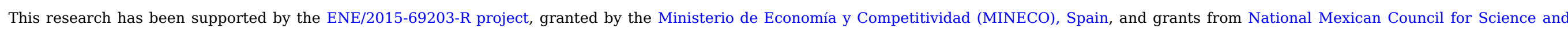

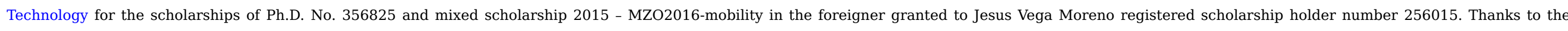

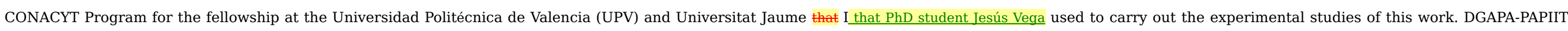
IG-100315.

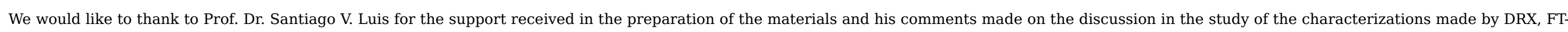
IR and SEM, Dr. Jose Antonio Irán Díaz Góngora for supporting the student’s stay, and Alberto López Vivas and Raúl Reyes Ortíz for their technical assistance.

\section{References}

[1] I. Dincer and M. Rosen, Sustainability aspects of hydrogen and fuel cell systems, Energy for Sustainable Development 15, $2011,137$.

[2] E. Bakangura, L. Wu, L. Ge, Z. Yang and T. Xu, Mixed Matrix Proton Exchange Membranes for Fuel Cells: State of the Art and Perspectives, Progress in Polymer Science 57, $2016,103$.

[3] J.H. Kim, S.K. Kim, K. Namb and D.W. Kim, Composite proton conducting membranes based on Nafion and sulfonated SiO ${ }_{2}$ nanoparticles, Journal of Membrane Science 415, 2012, 696.

[4] M. Yoon, K. Suh, S. Natarajan and K. Kim, Proton Conduction in Metal-Organic Frameworks and Related Modularly Built Porous Solids, Angew. Chem. Int. Ed 52, $2013,2688$.

[5] A. Iulianelli and A. Basile, Sulfonated PEEK-based polymers in PEMFC and DMFC applications: A review, International Journal of Hydrogen Energy 37, $2012,15241$.

[6] L. Li, J. Zhang and Y. Wang, Sulfonated poly (ether ether ketone) membranes for direct methanol fuel cell, Journal of Membrane Science 226, $2003,159$.

[7] G. Xiaomin, Liu Yonghua and Li Jinlong, Review on Modification of Sulfonated Poly (ether-ether-ketone) Membranes Used as Proton Exchange Membranes, Materials Science 21 (4), 2015.

[8] S. Mollá and V. Compañ, Nanocomposites SPEEK-based membranes for Duirect Methanol Fuel Cells at intermediate temperatures, J. Membr. SCi. 492, $2015,123$.

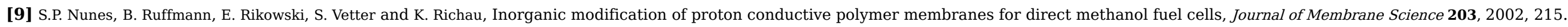

[10] M. Sadakiyo, T. Yamada and H. Kitagawa, Rational Designs for Highly Proton-Conductive Metal-Organic Frameworks, J. Am. Chem. Soc. 131, 2009 , 9906.

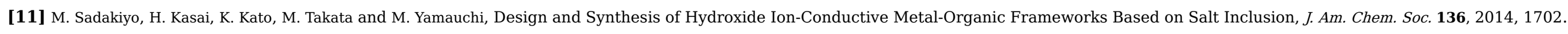

[12] C. McCarthy, V. Guerrero, G. Barnett and H. Jeong, Synthesis of Zeolitic Imidazolate Framework Films and Membranes with Controlled Microstructures, Langmuir 26, $2010,14636$.

[13] K.Y.A. Lin and H.A. Chang, Ultra-High Adsorption Capacity of Zeolitic Imidazole Framework-67 (ZIF-67) for Removal of Malachite Green From Water, Chemosphere 139, 2015, 624.

[14] J.B. James and Y.S. Lin, Kinetics of ZIF-8 Thermal Decomposition in Inert, Oxidizing, and Reducing Environments, J. Phys. Chem. C 120, 2016, 14015. 


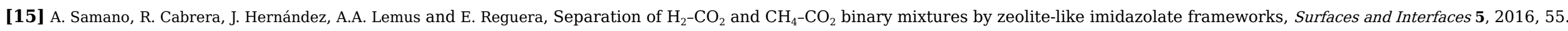

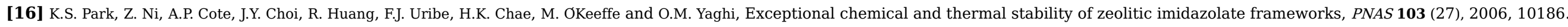

[17] R. Banerjee, A. Phan, B. Wang, C. Knobler and O.M. Yaghi, High-throughput synthesis of zeolitic imidazolate frameworks and application to $\mathrm{CO}_{2}$ capture, Science 319, $2008,939$.

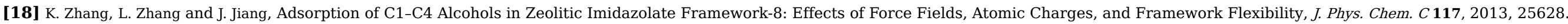

[19] K.S.W. Sing, D.H. Everett, R. Haul and I. Moscou, Reporting physisorption data for gas/solid systems, Pure and appl.chem. 54, $1982,2201$.

[20] C. Krause, J.R. Sangoro, C. Iacob and F. Kremer, Charge Transport and Dipolar Relaxations in Imidazolium-Based Ionic Liquids, J. Phys. Chem B. 114, 2010, 382.

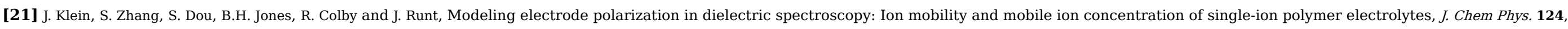
2006, 144903

[22] S. Tominaka and A.K. Cheetham, Intrinsic and extrinsic proton conductivity in metal-organic frameworks, RSC Advances 4, $2014,54382$.

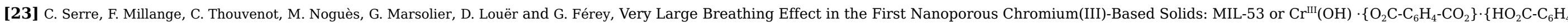
$\mathrm{CO}_{2} \mathrm{H}_{\mathrm{x}} \cdot \mathrm{H}_{2} \mathrm{O}_{\mathrm{y}}$ J. Am. Chem. Soc. 124 (45), 2002, 13519

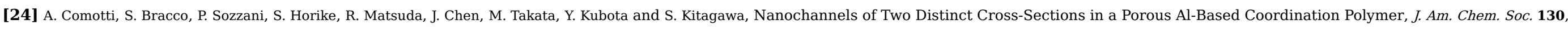
2008, 13664.

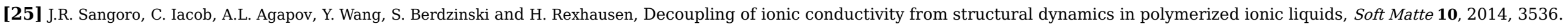

[26] S. Molla and V. Compañ, Polyvinyl alcohol nanofiber reinforced Nafion membranes for fuel cell applications, J. Membr. Sci. 372, $2011,191$.

[27] B.R. Matos, C.A. Goulart, E.I. Santiago and Muccillo, Proton conductivity of perfluorosulfonate ionomers at high temperature and high, Applied physics letters 104, $2014,091904$.

[28] A.K. Jonscher, A new understanding of the dielectric relaxation of solids, J. Materials Science. 16, 1981, 2037.

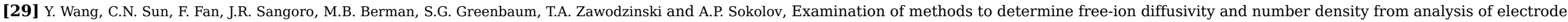
polarization, Physical Review, E 87, 2013, 042308.

[30] R. Coelho, Sur la relaxation d'une charge d'espace, Revue Phys. Appl. 18, 1983, 137

[31] J.R. MacDonald, Theory of ac Space-Charge Polarization Effects in Photoconductors, Semiconductors, and Electrolytes, Phys rev. 92, 1953, 4

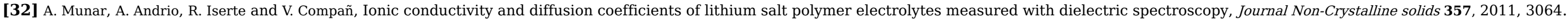

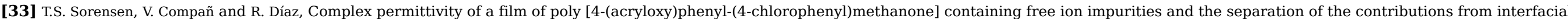
polarization, Maxwell-Wagner-Sillars effects and dielectric relaxations, J. Chem Soc. 92, 1996, 1947.

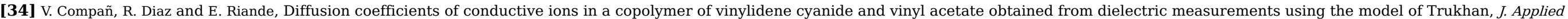
Physics. 79, 1996, 403.

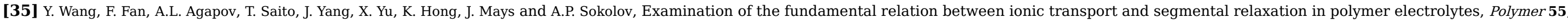
$2014,4067$.

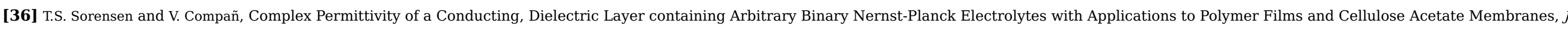
Chem Soc. Faraday Trans. 91, 1995, 4235.

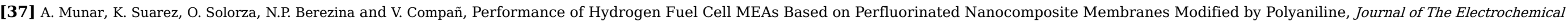


[38] T.A. Zawoddzinsk, M. Neeman, I.O. Sillerud and S. Gottesfeld, J. Phys. Chem. 95, 1991, 6040.

[39] A. Serghei, M. Tress, J.R. Sangoro and F. Kremer, Electrode polarization and charge transport at solid interfaces, Phys. Rev B 80, $2009,184301$.

[40] R. Murugaraj, AC conductivity and its scaling behavior in borate and bismuthate glasses, J. Mater. Sci. 42, 2007, 10065.

\section{Graphical abstract}

\section{Highlights}

- The ionic pair of tetrabutylammonium hydroxide was introduced within the Z8, Z67 and ZMix.

- Mixed matrix membrane of PEI/ZIFsT prepared using the modified ZIFs as fillers have been characterized.

- The relative amount of host molecules in Z8, Z67 and ZMix was determined.

- The local electric anisotropy characteristic of ZMix favors the amount of doping the TBAH salt.

- Activation energies for ZMixTW fillers and PZMixTW membranes were 0.13 and $0.14 \mathrm{eV}$, respectively.

\section{Queries and Answers}

Query: The author names have been tagged as given names and surnames (surnames are highlighted in teal color). Please confirm if they have been identified correctly. Answer: Yes

Query: Please check the presentation of all affiliations for correctness.

Answer: All the affiliations are correct, but if is possible change Física aplicada by Física Aplicada

Query: Please check the designated corresponding author e-mail address, and correct if necessary.

Answer: The corresponding author and e-mail address are correct

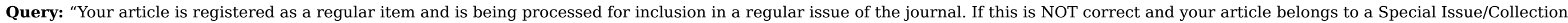
please contact a.elumalai@elsevier.com immediately prior to returning your corrections."

Answer: OKEY. The article is registered as a regular item and should be processed for inclusion in a regular issue of the journal.

Query: Please check the hierarchy of section headings.

Answer: Is correct. 
Query: Please note that Supplementary Figs. are cited in the text but not provided. Please provide Supplementary information or delete the citations from the text.

Answer: In attached file we have included the supplementary information. I thought it was saved from the previous version and did not need to re-include it

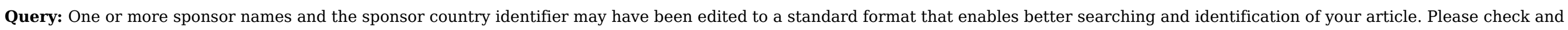
correct if necessary.

Answer: All the sponsor names and country identifications are correct.

Query: Please check the presentation of Tables 1 and 3, and correct if necessary.

Answer: Please, If it is possible to place the values in the columns of the tables 1, 2 and 3 in a centered way. ( See files attached. 